\title{
EDITORIAL
}

\section{Spirituality and Medicine: Science and Practice}

\author{
Richard J. Davidson, PbD
}

Waisman Laboratory for Brain Imaging and Behavior, Laboratory for Affective Neuroscience, and Center for Creating a Healthy Mind, University of Wisconsin-Madison

Ann Fam Med 2008:6;388-389. DOI: 10.1370/afm.900.

$\mathrm{T}$ hree articles in the current issue of the Annals explore different aspects of the role of spirituality in primary care. The article by Daaleman and colleagues explores the role of spiritual care at the end of life, a topic of critical importance for compassionate and humane treatment at this very sensitive period for the patient and his or her family. ${ }^{1}$ The article by Anandarajah describes several conceptual models for understanding the spiritual dimension in medical care with an emphasis on multicultural factors. ${ }^{2}$ The article by Katerndahl reports on an empirical study of the impact of knowledge of spiritual symptoms assessed by a self-report measure that includes such items as "peaceful," "a reason for living," and "sense of purpose" on health-care utilization, extreme use of services, and life satisfaction among primary care patients. ${ }^{3}$

Each article underscores the value of the primary care physician being attentive to the spiritual dimension of a patient's life context and taking this dimension into account in providing care. The body of work reviewed in these articles also draws attention to the importance of the primary care clinician embodying certain qualities that facilitate attention to the spiritual dimension of patients' care, including the notion of being present. This type of sensitive attention conveyed by the clinician is marked by physical proximity and intentionality. ${ }^{1}$ A central theme that cuts

Conflict of interest: none reported

\section{CORRESPONDING AUTHOR}

Richard J. Davidson, PhD

University of Wisconsin-Madison

Waisman Laboratory for Brain Imaging and Behavior 1500 Highland Ave

Madison, WI 53705

rjdavids@wisc.edu across all of these articles is that good medical care should include sensitivity to the spiritual dimension of patients' lives.

Several key questions raised by these articles are scientifically and clinically important and have major implications for how we train health care clinicians. At the definitional level, how can we objectively measure clinician sensitivity to spiritual dimensions of the patient? How can we assess such qualities as "being present"? How does the presence of these qualities in a clinician affect the patient? Are there changes in the patient beyond the subjective changes in well-being and perceived care? Can these qualities in the health care clinician be trained? These questions address some of the many important issues raised by these articles. The reality of these unanswered and complex questions should not detract from the remarkable fact that these questions are being raised and addressed in serious mainstream primary care medicine. The medical profession has become increasingly open to considering the importance of these issues for good medical care, a hopeful sign of a more complete and open view of the complex interdependence between health care clinician and patient, and within the patient, between the mind and body.

During the past decade, there have been great advances in our understanding of mind-brain relations and, in turn, relations between the brain and peripheral biologic systems that are important for health and illness. The study of mind-body relations is no longer just a closed black box. Science has begun to dissect the complex mechanisms by which the brain can influence peripheral biology, thereby providing the beginnings of a mechanistic understanding of how the mind may influence bodily systems. Moreover, in so far as our interpersonal environment, particularly the relationship between doctor and patient, can influence the patient's emotions, psychological state, and 
general level of well-being, the doctor is influencing the patient's brain and, through such influence, can affect peripheral biologic systems that are important for health.

There are many examples of biologic consequences of interpersonal relationships. In one recent study from my laboratory, we brought into the laboratory married couples who reported a highly satisfied married life. ${ }^{4}$ We then scanned the wife using functional magnetic resonance imaging of the brain while we induced anxiety through the threat of electric shock. In one condition, the wife held the hand of her partner, in another condition she held the hand of a stranger, and in a third condition she was alone. We found that activation of the pain matrix in the brain (a well-characterized circuit involving several interconnected structures including the anterior cingulate cortex, insula, prefrontal cortex, and hypothalamus) was significantly diminished when she was holding the hand of her spouse. Further, the more intimate and close the relationship was reported to be, the greater was the attenuation in neural activation in several of these brain regions. This study illustrates how high-quality social relationships can "get under the skin" and affect brain function in specific, predicable, and beneficial ways.

Two of the articles address the spiritual qualities of the healer, including compassion, presence, true listening, and others. Anandarajah notes interventions that include the embodiment of these qualities "...do not require inquiring about specific beliefs and take no more time than a clinician's usual duties. Rather, they require that health care professionals augment their everyday activities with presence, compassion, and positive intention." ${ }^{2}$ But augmenting everyday activities with these qualities requires practice, and it is here that the emerging discipline of contemplative neuroscience is highly relevant. ${ }^{5}$ Contemplative neuroscience is focused on changes in brain function and structure that come about as a function of contemplative practice. It is predicated on the large body of literature on neuroplasticity, which holds that the brain is the key organ built to change in response to experience and training. One core element we have learned from this emerging literature is that practice is required to change the brain and that more practice leads to more pronounced transformation, at least for some brain circuits that underlie compassion and attention. A key implication of this work is that opportunities be provided to the primary care physician and other health caregivers to undergo contemplative training to culti- vate these universal qualities, such as sensitive attention, compassion, and positive intention.

As noted earlier, the brain circuits transformed by meditation play a key role in modulating peripheral biologic systems that may be consequential for health. For example, in a study of an 8-week mindfulnessbased stress reduction intervention, we found that brain activity was altered and that the magnitude of change in brain activity predicted the magnitude of increase in antibody titers to an influenza vaccine. ${ }^{6}$ In other basic research on mind-brain-body interaction not involving meditation, we found that some of the same brain circuits affected by meditation are those implicated in modulating inflammatory processes in the lung in asthmatics. ${ }^{7}$ If these circuits can be influenced by compassionate care, they may have downstream impact on peripheral biologic systems that might affect health and illness.

As we move forward with this important area in the future, it is essential that future studies of spiritual care incorporate rigorous biologic measures to determine the relation between caregiver characteristics and clinician-patient interaction and direct biologic measures that are relevant to disease outcome. Such mechanistic studies will help the wider biomedical community to understand and appreciate the import of spiritual qualities in medical care

To read or post commentaries in response to this article, see it online at http://www.annfammed.org/cgi/content/full/6/5/388.

Key words: Spirituality; mind-body relations (physiology); physicianpatient relations

Submitted August 11, 2008; accepted August 11, 2008.

\section{References}

1. Daaleman TP, Usher BM, Williams SW, Rawlings J, Hanson LC. An exploratory study of spiritual care at the end of life. Ann Fam Med. 2008;6(5):406-411.

2. Anandarajah G. The $3 \mathrm{H}$ and BMSEST models for spirituality in multicultural whole-person medicine. Ann Fam Med. 2008;6(5):448-458.

3. Katerndahl DA. Impact of spiritual symptoms and their interactions on health services and life satisfaction. Ann Fam Med. 2008;6(5): 412-420.

4. Coan JA, Schaefer HS, Davidson RJ. Lending a hand: social regulation of the neural response to threat. Psychol Sci. 2006;17(12):1032-1039.

5. Davidson RJ, Lutz A. Buddha's brain: neuroplasticity and meditation. IEEE Signal Processing. 2008;25(1):171-174.

6. Davidson RJ, Kabat-Zinn J, Schumacher J, et al. Alterations in brain and immune function produced by mindfulness meditation. Psychosom Med. 2003;65(4):564-570.

7. Rosenkranz MA, Busse WW, Johnstone T, et al. Neural circuitry underlying the interaction between emotion and asthma symptom exacerbation. Proc Natl Acad Sci USA. 2005;102(37):13319-13324. 